\title{
Theory and Practice of Aging during the COVID-19 Pandemic
}

\author{
A. G. Golubev ${ }^{a, *}$ and A. V. Sidorenko ${ }^{b, * *}$ \\ ${ }^{a}$ Petrov National Medical Research Oncology Center, Pesochnyi Settlement, St. Petersburg, 197758 Russia \\ ${ }^{b}$ European Center for Social Welfare Policy and Research, Vienna, 1090 Austria \\ *e-mail:lxglbv@rambler.ru \\ **e-mail: sidorenko.alexandre@gmail.com \\ Received May 18, 2020; revised May 19, 2020; accepted May 20, 2020
}

\begin{abstract}
Never before in history has population aging been a driving factor in epidemics to the same extent as with the current COVID-19 pandemic, with its dramatic shift in mortality towards older age groups. The paper presents the results of an analysis of the COVID-19-related mortality data for Spain, Italy, and Sweden, which show that within the 30- to 90-year age range, the logarithms of mortality rate depend on age linearly, and all regression lines are strictly parallel to the lines corresponding to the dependencies of the general mortality on age in accordance with the Gompertz law. In all cases, irrespective of the countries and epidemic stages, the mortality doubling times within this age range are close to 7.5 years. The probabilities of infection with the SARS-CoV-2 coronavirus, the causative agent of COVID-19, and of the development of the clinical symptoms of infection depend on age to a much lesser extent. Based on these observations, three main points are proposed for discussion: (1) Older people have become the main victims not only of SARS-CoV-2 itself but also of the measures undertaken to prevent its spread; (2) At the same time, older people are not the main force driving the spread of SARS-CoV-2, and (3) Older people can and should participate in the fight against the pandemic and in overcoming its consequences, but not through their selective isolation and other forms of discrimination. People over 65 years of age make up a considerable segment of the population and have at least as much right as other age groups to have their needs and interests be respected and observed, including the right to as high quality of life as is accessible even in extreme situations. The prospects for full control over SARS-CoV-2 are vague. This is why those who are in charge of decisions that concern people over 65 years of age should mind that, unlike the situation in the Middle Ages, the age of $65+$ is the individual future of almost everyone.
\end{abstract}

Keywords: aging, gerontology, geriatrics, COVID-19, epidemiology

DOI: $10.1134 /$ S2079057020040062

\{Epidemics like the coronavirus outbreak are a mirror for humanity, reflecting the moral relationships that people have toward one other.\} Frank M. Snowden https://www.facebook.com/newyorker/posts/epidemicslike-the-coronavirus-outbreak-are-a-mirror-forhumanity-reflecting-the/10157255100133869/

\{...The Government of the Russian Federation, together with the top officials (heads of top executive governmental bodies) of the constituent entities of the Russian Federation, should ensure... that a self-isolation regime is maintained for persons over 65 years of age. $\}$ http://kremlin.ru/events/president/news/63358

\section{INTRODUCTION}

At the very start of the COVID-19 infectious disease pandemic (CoronaVirusDisease 2019), attention was drawn to the fact that the age structure of mortality caused by its pathogen, the SARS-CoV-2 (Severe Acute Respiratory Syndrome Coronavirus 2) corona- virus, is characterized by a significant shift towards older age groups (Fig. 1).

Reports concerning the COVID-19 pandemic are now proliferating mainly in the form of preprints, i.e., almost without peer review. An analysis and summary of these reports are also challenging due to the significant differences between countries in the approaches, methods, and assessment criteria for epidemiological indices [3], as well as their position regarding prevention measures, including social distancing in the form of voluntary/recommended self-isolation or forced/mandatory strict isolation of different population groups. However, there is a perspective from which the most diverse data fits into a single model. If the relationship between, on the one hand, the quotients obtained by dividing the numbers of deaths attributed to the coronavirus within certain age intervals by the numbers of the reported cases of coronavirus infection within the same intervals and, on the other hand, the average ages in those intervals is presented on a semilogarithmic scale, then straight lines 


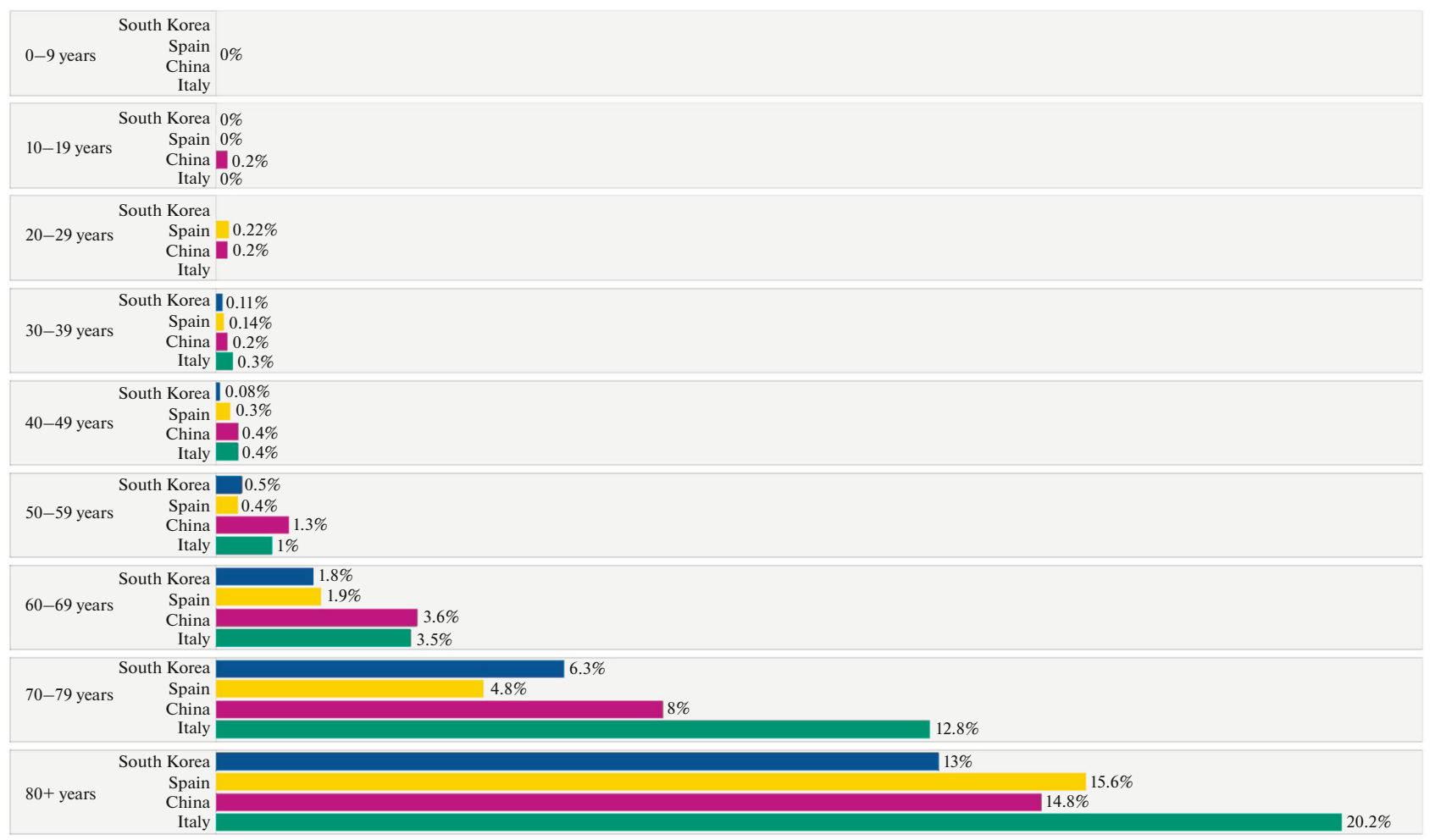

Fig. 1. Example of data demonstrating the relationship between the COVID-19 mortality rate and age (http://ourworldindata.org/mortality-risk-covid?county).

that are strictly parallel to each other and to the line representing general mortality dependence on age will be obtained in all cases (Fig. 2).

The linear dependency between the logarithm of mortality rate and age is equivalent to the exponential increase in the death rate with age, which is known in gerontology as the Gompertz law (or model):

$$
\mu(t)=\mu_{0} \mathrm{e}^{\gamma t} \text { or } \ln \mu=\ln \mu_{0}+\gamma t .
$$

The factor $\gamma$ at age $t$ is usually interpreted as the average aging rate for the population where the quantitative estimates of the model parameters are determined. Another way to present aging rate, if it leads to an exponential increase in the death rate, can be the mortality doubling time $d=\ln 2 / \gamma$. For all (!) data in Fig. $2, d \approx 7.5$ years.

There are numerous publications on the feasibility and applicability of the Gompertz law (or model) to the relationship between age and the risk of death (e.g., $[14,15]$, which provide references to different points of view). As for Fig. 2, the application of this model to the analysis of COVID-19 mortality data indicates that there is some order in the world, even in a situation close to chaos, as long as the model indeed reflects this order. Figure 2 shows a strikingly consistent pattern that remains the same at different epidemic stages, from the exponential growth phase to the plateau (data for Spain), and in different countries that vary greatly in their approaches to controlling the epidemic and the dynamics of its development, from Sweden to Italy. It should be emphasized that we refer here only to the linearity and parallelism of the plots in Fig. 2 and not to the extent to which they are shifted relative to the total mortality rate. It is these shifts that depend on the case-recording methods used in different countries and on the stages of the epidemics.

In all cases, the invariant mortality doubling time, which is close to 7.5 years, means that the risk of death among all patients with infection symptoms is by approximately $2^{(70-30)} / 7.5 \approx 40$ times higher for those aged 70 than for those aged 30 . The risk of death is generally about $8 \%$ in the 60 - to 70 -year-old group and is higher in men than in women (10 and 6\%, respectively, data not presented).

Similar observations, as well as the data for China and South Korea, laid basis for the conclusion that COVID-19 can be classified as an emergent disease of aging [31] that appeared in addition to the diseases typically prevalent in older people, mostly chronic noninfectious diseases, including primarily all cardiovascular and neurodegenerative diseases and cancer.

What is critically important is that only $2 \%$ of COVID-19 victims do not have any concomitant diseases typical of the older age, while the remaining $98 \%$ suffer primarily from such diseases as hypertension, coronary heart disease (CHD), and type-II diabetes 
mellitus, which often develop in combination in a single patient [31]. It is known that the number of patients with these newly diagnosed diseases and the rate of mortality caused by them increase exponentially with age, doubling time being ca. 6-8 years. The presence of each of these diseases increases the risk of death from any cause, including infections.

Interestingly, it is also known for other types of pneumonia that hospitalization rates and mortality rates caused by them increase exponentially with age, doubling times being the same [18, 28], as was confirmed by Santesmasses et al. [31]. Thus, almost any pneumonia can be classified as a disease of aging on the same grounds.

Moreover, the prevalence of some other infections that normally do not affect lungs also shows nearly exponential growth [28]. This relates to the infectious diseases caused by Haemophilus influenzae, streptococci, West Nile virus, and influenza A virus, etc. Obviously, the proportion of patients suffering from typical age-related diseases will be higher among infected older age individuals.

The 1917-1919 Spanish flu pandemic caused by the influenza A virus holds an exceptional position in this regard. The peak of mortality at that time was observed in the middle age group. This is still often considered typical of acute respiratory disease epidemics, and, if judged from this perspective, the current coronavirus pandemic appears to be exceptional. However, it can be seen that, in terms of the increase in mortality with age, the pandemic is only exceptional quantitatively, not qualitatively, and once again confirms that the ability to withstand any cause of death decreases as we get older.

The discussion of the reasons why the organism's ability to withstand the development of any disease or death from a wide variety of causes decreases with increasing age and is associated with an exponential increase in mortality goes far beyond the scope of this article. The authors' positions regarding these issues can be found elsewhere [1,2]. Still, there are two points which are worth noting.

The special role of hypertension as a concomitant disease in increasing the risk of SARS-CoV-2 infection fatality draws attention to the possible involvement of the cellular protein that SARS-CoV-2 "clutches"-the angiotensin-converting enzyme 2, which participates in blood pressure regulation. Its expression in tissues increases with age and in agingassociated diseases [12, 31].

Another point is the status of the immune system. Aging is associated with involution of the thymus, the organ where lymphocytes capable of producing an immune response to antigens encountered for the first time undergo differentiation. This involution takes place in virtually all mammals. Its rate is higher in short-living than in long-living animals. The reasons underlying the emergence and fixing of this phenom-

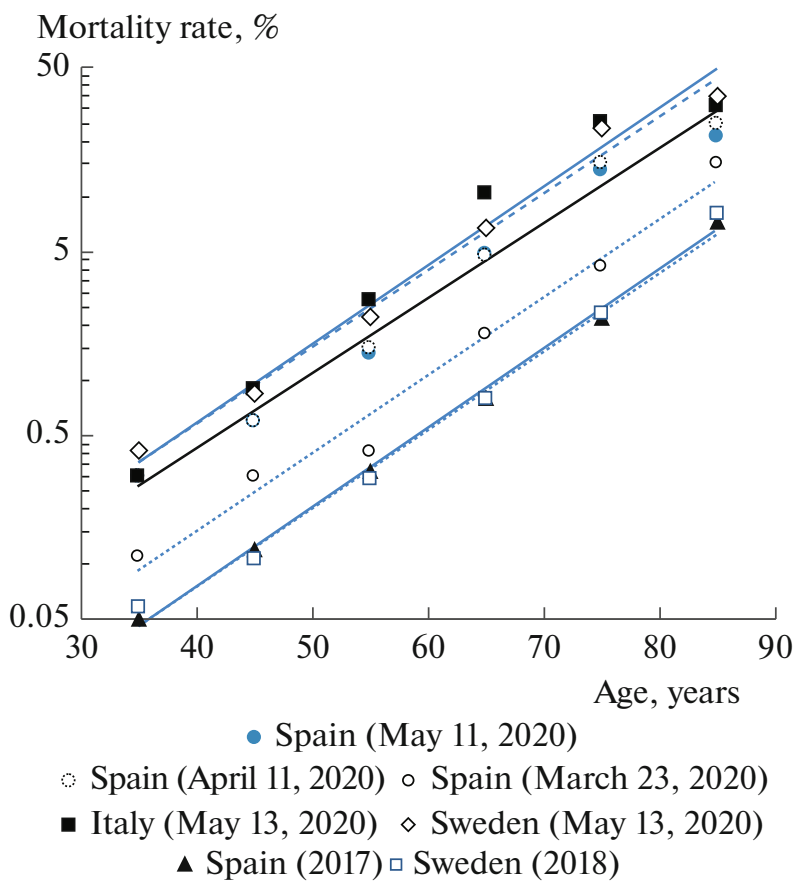

Fig. 2. Dependencies of mortality rates on age (on a semilogarithmic scale). Case fatality rates in COVID-19 patients:

-in Italy (fine line); data source https://www.epicentro.iss.it/en/coronavirus/bollettino/Infografica_15aprile\% 20ENG.pdf;

-in Sweden (dashed line); data source https://www.statista.com/statistics/1107913/number-of-coronavirus-deaths-in-swedenby-age-groups/;

-in Spain, at the three epidemic stages; data source https://www.isciii.es/QueHacemos/Servicios/VigilanciaSaludPublicaRENAVE/EnfermedadesTransmisibles/Paginas/Informes-Previos-COVID-19-sanitarios.aspx. The plots for the dates May 11 and April 23 (dotted and dashed lines) coincide. Total mortality (\%) during a year according to http://mortality.org data. The plots obtained for Sweden and Italy almost coincide.

Dots are set against the centers of the 10-year age intervals from $x 0$ to $x 9$ years.

enon in evolution are unclear. As for its consequences, it is thought that thymic involution may form the general bases for a decrease in the organism's ability to resist both infectious diseases and cancer [28]. Additionally, the aging immune system becomes increasingly loaded with cells that retain memory of antigens met earlier [27]. This gradually makes the immune system less capable of learning. The immune system of older people, already trained to combat the infections that constantly circulate in the population, allows older individuals to more efficiently defend themselves from such infections as compared to young people with an untrained immunity. However, new or rapidly mutating infectious agents are more dangerous for older individuals, and vaccine treatment can be less efficient in this age group [23]. This fact is directly related to the prospects for the suppression of SARS$\mathrm{CoV}-2$ circulation in human populations. In the long- 


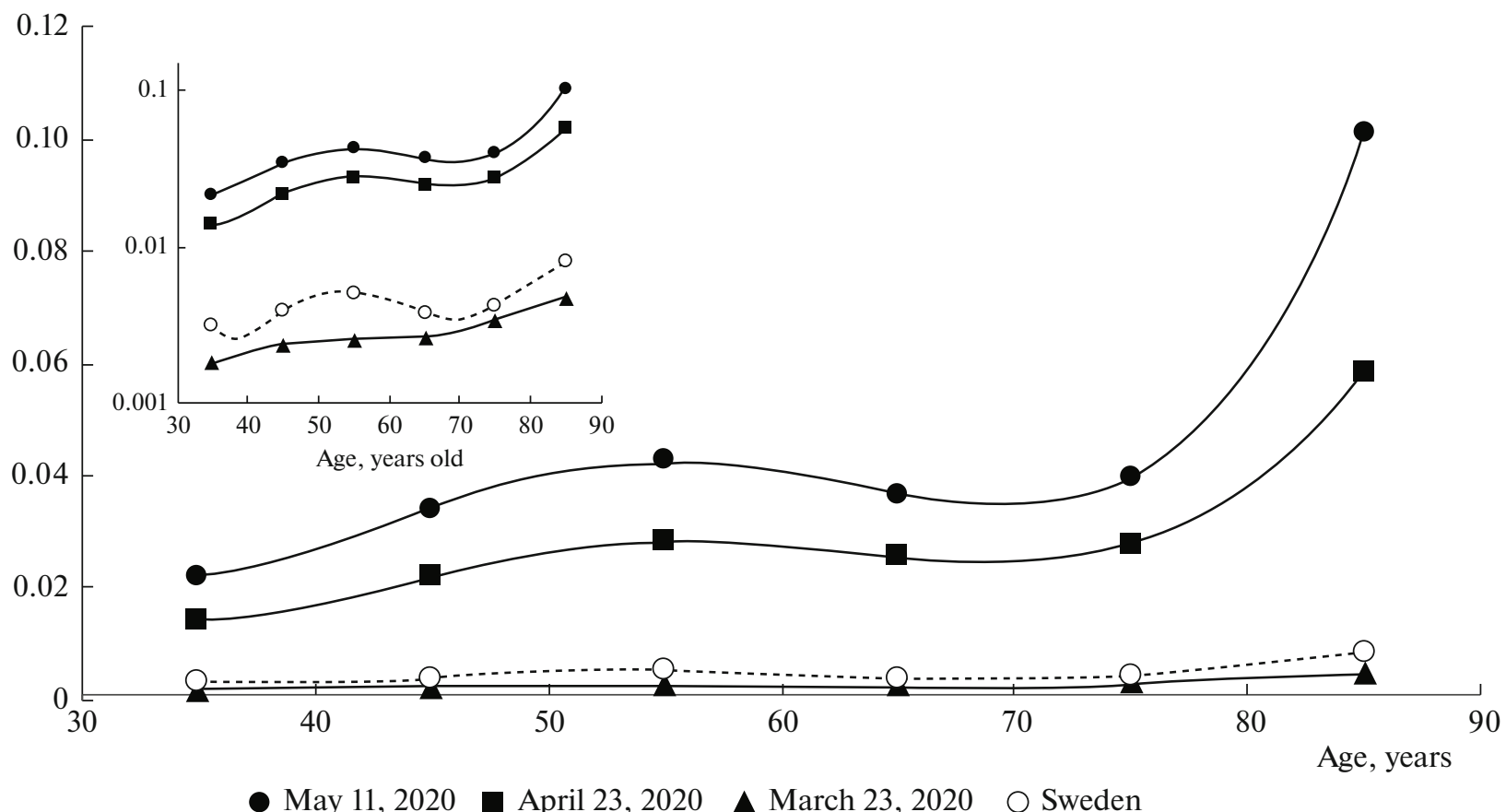

Fig. 3. Age dependencies (dots are set against the centers of the ten-year age intervals) for the relative number of diagnosed COVID-19 cases in Spain at the three different stages of epidemic and in Sweden as of May 13, 2020.

The inset provides the same plots on a semilogarithmic scale. The COVID-19 data sources are the same as in Fig. 2. The sizes of populations within the 10 -year age intervals in 2018 were calculated based on data available in the Human Mortality Database (http://mortality.org).

term, it may happen that COVID-19 will remain the cause of new epidemic outbreaks while a certain portion of individuals, including those aged 65 years and older, demonstrate insufficient immunity against this virus.

It should also be noted that the state of the immune system determines not only the ability of the organism to resist an infection and, consequently, lower the mortality rates among the infected individuals but also the probability of the infection as such, which is expressed as the number of infected individuals within a certain age interval per the total number of individuals within this age interval. However, the age dependencies of mortality calculated per the number of diagnosed cases, of the number of such cases and of the real risk of infection do not necessarily match one another.

The dependency of the probability of COVID-19 diagnosis on age can be deduced from the comparison of the number of registered infected individuals and the number of individuals in each given age range (Fig. 3).

As is the case with Fig. 2, it is important to emphasize that the key point here is the age dependencies, not the absolute values, which depend on the epidemic stage and the country (as far as different countries practice different approaches to the control of COVID-19). Despite all of the differences, it can be seen that the number of diagnoses per the number of "those at risk of diagnosis" depends little on age within the range of 45-75 years; a small peak being observed at around 50-55 years of age. What are the grounds in this case for the total isolation of all individuals aged $65+$ ?

As for the absolute numbers, it is clear that the number of diagnoses in Spain increased in all age groups as the epidemic developed, and the situation in Sweden is determined not only by the actual infection rate in the population but also by the adopted practice of COVID-19 diagnosis biased to severe cases. However, it should be emphasized here once again that the pattern of age-related changes does not depend on the time or place.

In order to get an idea of the actual dependencies of the risk of infection and the number of SARS-CoV-2 infected individuals, including asymptomatic cases, on age, the results of tests in the entire population, or at least in a sufficiently large random sample, at the stationary phase of the epidemic should be obtained. Such studies, which use serological antibody tests for SARS-CoV-2, are already underway or are planned in many countries, but they are still rarely published.

In the flow of papers promptly posted on servers like MedRxiv and BioRxiv, only two reports could be found $[11,33]$ to present the results of the studies of random sera samples collected irrespective of indications for testing for SARS-CoV-2 antibodies and binned into defined age intervals. 

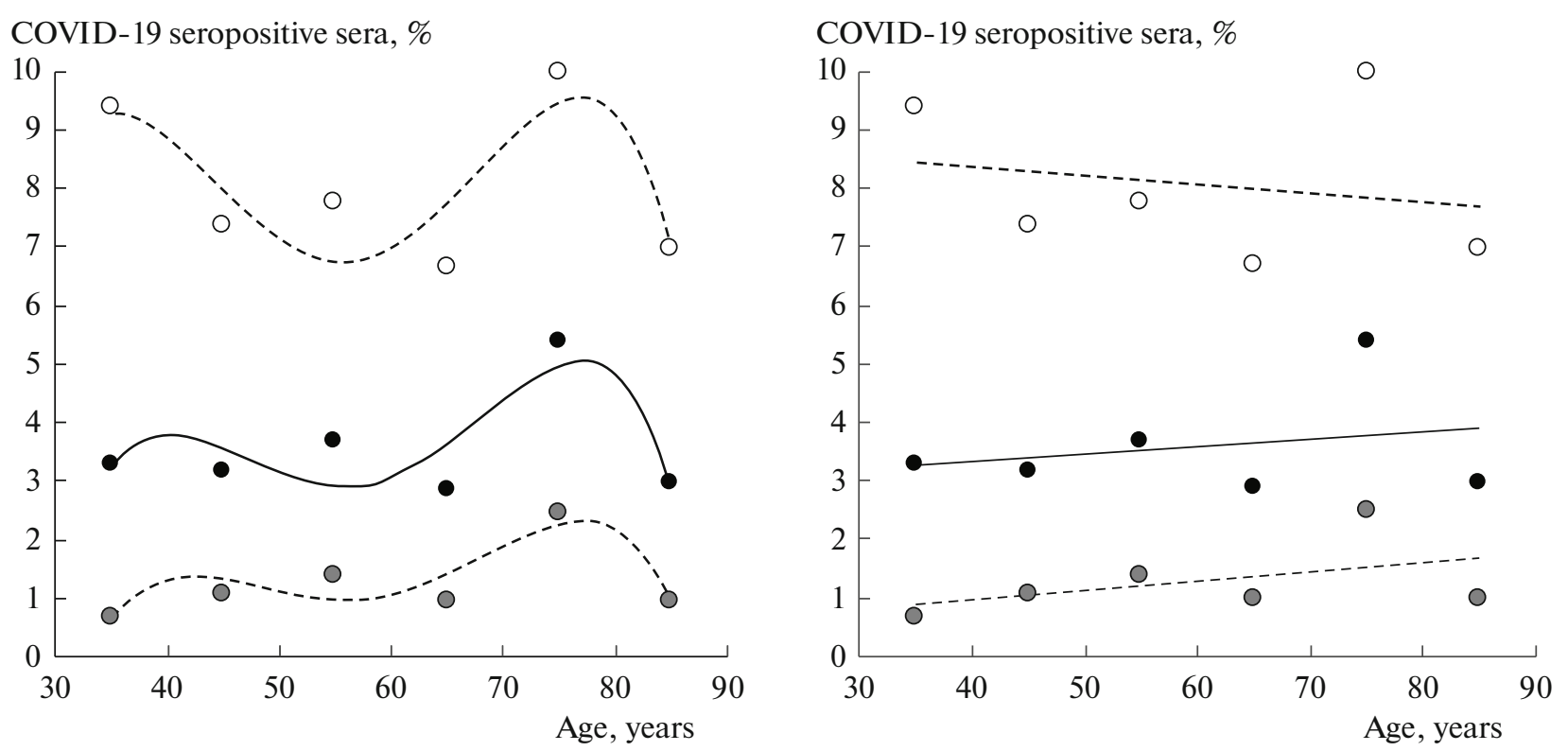

$\longrightarrow \% \quad--0--+95 \%$ CI $\quad--0--95 \%$ CI

Fig. 4. Dependence of the proportion of COVID-19 seropositive sera on the blood-donor age in Kobe (Japan). The data on men and women reported in [11] are combined. To the left, trend lines are presented as fourth-order polynomial regressions; to the right, linear trends are shown. In both cases, the 95\% CI is indicated above and below the lines.

In a work reported by Japanese authors [11], the data for Kobe are binned in 10-year age intervals. The simplest calculation made with these data, however limited they are, in no way shows that the risk of infection increases with age as sharply as the risk of lethal complications of the infection (Fig. 4).

Obviously, the behavioral differences between the age groups should not be ignored. However, even in this case, the same considerable increase in the risk of infection upon an increase in the age from 35 years to 70 years, as is observed with the risk of death after infection, can not be demonstrated. The data obtained in Gilan Province, Iran were stratified by age less precisely, namely, $18-60$ years old and above 60 years of age. The percentage of SARS-CoV-2 seropositive individuals were $21 \%$ (95\% CI, 14-30) and 26\% (95\% CI, 15-41), respectively. In this case again, no signs of any significant shift towards the older age can be observed.

However, it can be seen that the general SARSCoV-2 seropositivity rate is considerably higher than the estimates made based on selective testing when medically required. According to the calculations made by the Japanese authors, the number of individuals positive for SARS-Cov-2 in Kobe should be 400800 times higher than the number of cases confirmed by testing when medically required. These conclusions are somewhat shocking. However, from all appearances, this may prove to be a common case. According to [33], the situation in Iran is not much better. In
France, as follows from the results of epidemiological situation modeling [30], the number of SARS-CoV-2 seropositive individuals in some large regions may reach $10 \%$ of the population.

If the number of lethal cases is determined not vs. the registered COVID-19 cases (case fatality rates, $\mathrm{CRF}$ ) but rather vs. SARS-CoV-2 infection cases, then even the most rough calculations show that the rates of mortality caused by any SARS-CoV-2 virus infection, including asymptomatic cases (infection fatality rates, IFR) must be two orders of magnitude lower than the 6-10\% CFR. If it is about 400-800 times lower (see the above [11]), e.g., 500 times lower, then IFR appears to be $0.01-0.02 \%$. Is it high or low? It depends on what we compare. In Russia, the total per year mortality rates for the individuals aged 65 and 75 years are 1.5 and $4 \%$ in women and 4 and $8 \%$ in men, respectively (mortality.org). If we consider that the duration of COVID-19 from the moment of infection to the disease outcome is about 1 month, then it appears that IFR and the risk of death caused by any other factor during one month are quite comparable. COVID-19 increases mortality, which may also be due to other causes, by about two to three times at any age. Since the risk of death at 70 years of age is about 40 times higher than that at the age of 30 years (Fig. 1), the age-related differences in COVID-19 mortality will be approximately the same. The total mortality caused by SARS-CoV-2 in the population increases with an increasing number of older persons in the population. This will continue until everyone contracts 
the disease or until a total effective vaccination is carried out, i.e., until collective immunity is achieved or until effective therapeutic methods for the prevention of death from this disease are developed.

It is then reasonable to ask what pandemic-control measures would be the most promising in an aging society? In fact there are two questions: (1) what measures are needed "here and now," regardless of what may happen next; and (2) what measures are justified with the prospect of the permanent presence of SARSCoV-2? The latter scenario is quite possible [22], considering cases with influenza viruses and pneumococci and streptococci. If SARS-CoV-2 becomes a permanent presence, it should be born in mind that the proportion of individuals aged 65 years or older will further increase in the population (provided COVID-19 or self-isolation will not kill them immediately). In this view, a third question arises: is it wise to make decisions on the first two questions without consideration of the gerontological aspect of the situation?

In the long term, the only way to reduce the load on the healthcare system imposed by the diseases of aging, i.e., those for which the medical cost of treatment increases with age (and this can be seen to be true for COVID-19 as well), is to reduce the risk factors of these diseases. The main factor behind the need for resuscitation procedures in COVID-19 is not the age itself but the accompanying hypertension, CHD, obesity, and diabetes [12, 19,34, 36] or merely a decrease in glucose tolerance [40], which is possible at any age. These disorders can be classified in different combinations as metabolic syndrome, inflammaging, or another term. In any case, it is important that these disorders contribute to the development of the diseases, including cardiological, neurological, nephrological, and pulmonary diseases, and even cancer [17], which, in aggregate, as indicated above, cause mortality rates comparable with those caused by coronavirus during the pandemic.

The authors who have included, not without reason, COVID-19 among the diseases of aging [34] have concluded that this can pave the way for the use of future antiaging pharmacological products, such as senolytics, to prevent and treat potentially deadly infectious diseases. So, what? Does this mean that it is better for older people to stay at home until such drugs are developed? In the meantime, it becomes increasingly clear that the implementation of the effects of virtually all potential antiaging drugs involves only some of the several molecular mechanisms that together can decelerate the decline in the functional abilities and increase life expectancy when nutrition and physical activity are optimized (e.g., [16]). At the same time, population studies clearly demonstrate that adequate physical activity counteracts the development of almost all age-related diseases, including infections, more effectively than any of the substances tested in this regard [8, 10, 24, 37]. Adequate physical activity, which makes aging healthier and thus less burdensome for the society and people themselves, should be combined with active mental activity, abundance of experience, an optimistic view of life, and involvement in social relations [20, 21, 32, 39]. This observation is based not on just "everyday experience" but on the facts established in large-scale population studies and corresponding measurements and calculations. Then, should our limited resources be spent on the development of simulators of those measures that will surely produce the desired result of extending a healthy life expectancy, if those resources can be used on measures that may help to motivate and create conditions for a healthy lifestyle?

Meanwhile, the most stringent forms of self-isolation, which are justified in principle, are imposed on the 65+ part of the population. They are imposed in an indiscriminate way, regardless of the actual health status of people aged 65+, many of whom may at the moment be much healthier than some people in their forties or even younger. These older people are still healthy only because of their personal attention to factors that reduce mortality from age-related diseases, including COVID-19 complications, the factors now being nullified by self-isolation measures. At the same time, each day of the rest of lifetime means a lot more for those who have reached $65+$ than for those who still have time to make up for losses. How is this borderline justified at all-at 64 you are allowed, but at 66 you are not?

The reason of the "super-mortality" of older people in the context of the COVID-19 pandemic is not their age itself but their health conditions [5]. One might ask, of course, who would individually distinguish more or less healthy people under extreme conditions? However, why not entrust the informed decisions on this issue to those who are the most concerned ones? It has been noted and repeatedly stated that the success in the fight against pandemics depends on the mutual trust among all parties concerned [9]. Absolutely, on the mutual trust. It is love that may be one-way, but respect and trust can only be mutual.

How and to what extent can it be justified that social distancing, which in the context of pandemics is justified in principle, would require, among other things, that forced restrictions, the so-called "recommendations," the failure to comply with which is sanctioned by a fine that grows with the age of the violators, should be the most severe towards older people? At the same time, it is older people who take the most responsible attitude towards their health and readily follow reasonable antiepidemic recommendations, such as masks and gloves in public places and the observance of personal space and hygiene, and refrain from participation in mass gatherings, including joint barbecues, as well as demonstrative protests for their own sake. 
However, if one looks beyond the sentimental screen, it is easy to see that the only purpose of age discrimination, which is already becoming apartheid, is to reduce the competition for the limited number of intensive care beds between those who are less likely to have fewer extra years of less productive life and those who are more likely to have more extra years of more productive life.

An impartial discussion of the appropriateness, acceptable boundaries, and publicly stated justifications for this kind of "optimization" of public resource allocation between different age groups may be found in the literature [13]. The remote consequences of these "optimization" measures, which may be manifested in increased mortality-well, let it be mortality, but not overloaded healthcare facilities-are also discussed, since self-isolation nullifies all of the consequences of a healthy and active lifestyle, which prevents the development of diseases associated with aging, including complications from coronavirus infection [4].

No one really knows what the course of events will be like in pandemics. The significance of the experience gained in previous pandemics is largely limited by the very fact that the factor of aging has never been so significant previously. The lack of real experience that would facilitate decision-making is made up for by the development of models and the playing out of different scenarios with these models. The number of published models has already reached several dozen, if not more than a hundred. Of these, only one (!) took the age aspect of the situation into account [29]. The variants of social distancing measures used in different ways in each of the three age groups (the young, middle, and older ages) were simulated based on data obtained in France. The conclusion made by the authors is intentionally presented here verbatim, so as not to distort any semantic nuances:

$\{$ We found that epidemic suppression can only be achieved by targeting isolation of young and middle age groups with high efficiency. All other strategies tested result in a flatter epidemic curve, with outcomes in (e.g. mortality and health system overcapacity) dependent of the age groups targeted and the isolation efficiency. Targeting only the elderly can decrease the expected mortality burden, but in proportions lower than more integrative strategies involving several age groups... The only partial lockdown strategy that achieved suppression in our simulations was targeting young and middle ages at efficiencies higher than $60 \%$. The impact of this strategy on the outcomes assessed (epidemic duration, death burden, days over capacity and peak of severe cases) was comparable to a complete lockdown at an efficiency higher than $40 \%$, because these age groups (young and middle ages) contribute greatly to transmission...Alternatively, targeting only the elderly population could theoretically decrease the death toll in similar proportions than if combined with another age group... However, relying just on targeting the elderly would result in thousands more severe cases at the peak of the epidemic than for other strategies, and the health system could be over capacity for several weeks. The consequences of this could be offset if current efforts to discover effective treatments and to rapidly increase the country's bed capacity at intensive care units are successful.\}

The content of the cited article shows that the social isolation of older age groups makes the smallest contribution to the overall effectiveness of antiepidemiological tactics, which means that these measures may be relaxed to the greatest possible extent, while their reinforcement is justified at the lowest level. Of course, the conclusions made based on a model are predetermined by the model's starting points. Any of them may be challenged in any model. "Essentially all models are wrong, but some are useful" [6]. How useful is this one? France, where the practice of keeping older people in nursing homes is widespread, is very different from Russia in this respect and in terms of the age structure of the population in general. Furthermore, "acceptable" is one thing, but the extent to which it is reasonable and justified is a different thing. How far may "optimization" go? Not only in terms of tactics, but also in terms of strategy. All of these considerations may be declared to be subtleties, which there is currently no time to consider. This is true if the only available tool is a hammer. Then, yes, the only thing that may be done is treating all around like nails.

It is also important to recall that the difficult situation faced by Russia's healthcare system in the context of the pandemics was only exacerbated by the "optimization" measures undertaken earlier by "efficient managers," those who are primarily interested in the fulfillment of the immediate economic optimization criteria. "Lawyers, economists, and managers are too preoccupied with lawmaking and legal obedience to pay attention to the regularities that exist independent of their consciousness and beyond their accessibility" [1]. All of this contributes in no way to the mutual trust and respect (see above) between the managers and managed subjects. One can also recollect the wellknown experiment on the optimization of financial and economic relations between different age groups, which was carried out in "Crime and Punishement" by Rodion Raskolnikov, who was a law school dropout but still a lawyer by training.

Let us see what an "epidemics mirror" can show us in this respect. Looking into it, those who have reached the age of 65+ may at last understand who they are from the economic, legal, and managerial point of view. They will see that they should not live, because it could lead to increased competition with the more promising part of the population for hospital beds, and they should not die either, because it will increase the load on the civil registry and funeral services. Those who lingered in relieving the managers involved in the optimization from such extra trouble 
are simply too late to be late. Staying at home is the destiny of those $65+$ years old.

There is also another attitude to the current situation: the coronavirus pandemic is an attempt made by nature or some other force to improve the demographic situation in countries with a high percentage of older people and to rejuvenate the countries' population. In the scientific literature, if these ideas are ever discussed, it is only as a reaction to their appearance in the mass media. Consider the term "boomer remover" (https://nypost.com/2020/03/19/ morbid-boomerremover-coronavirus-meme-onlymakes-millennials-seem-more-awful/) as an example. Remover is a savior, and Baby Boomer is one who was born during the childbearing boom around 1946-1964. Today, this population group, those about 70 years old, is still numerically large, and it is these people who hold influential positions in politics and economics. The positions and views of these people are not always clear, or are even annoying, to those whose life attitudes are determined by the ideology and practice of digital technologies. In times of crisis, conspiracy theories flourish rapidly, driving the foundations of social life that have developed over centuries, including humanism, human rights, long-term economic feasibility, and, finally, common sense, out of people's mind.

The UN "Convention on the Prevention and Punishment of the Crime of Genocide" (https://treaties.un.org/doc/publication/unts/volume\%2078/volume-78-i-1021-english.pdf) defines one form of genocide as "the deliberate infliction on the group conditions of life calculated to bring about its physical destruction in whole or in part." It is hard to imagine that someone somewhere would implement measures to reduce the number of older people, considering them overly burdensome during pandemics. However, unintentional "gerocide" may be based on the negligence of the interests and rights of these people.

It is hardly reasonable to regard the trends detected in the halls of power as the manifestation of disposition towards genocide in the form of "gerocide." However, one can also recall the term "ageism," which was suggested by the outstanding American gerontologist Robert Butler in the late 1960s. His article [7] has been cited in more than 2000 publications, including the 2020 review [25]. The term ageism refers to the systematic application of negative stereotypes to older people and discrimination against them. It is easy to find similarities between ageism and other forms of discrimination, such as discrimination by race, or racism, and by gender, or sexism. Butler saw the roots of negative stereotypes about old age and of ageism in the personal distaste of the young and middle-aged people for aging, illness, and disability and in their fear of their future inevitable debility, "uselessness," and death.
Ageism during pandemics may show itself in the form of accusations and discrimination against older people based on the suspicion that they may be a serious disease spread factor and a source of unnecessary problems. There is no basis for suspicions of this kind. As indicated above, older people are the main victims not only of the infection itself but also of the measures aimed to control it, but they are in no way the main agent of its spread. Of course, infected older people may become a source of infection for other people, which is possible if they fail to observe isolation and thoughtlessly participate in public events, including religious ones. At the height of pandemics, ageism may show itself in restricting older patients' access to limited medical resources, such as ALV machines. Such restrictions based only on the patient's age have been noted by the UN human-rights experts [38].

The preservation of life and health of people of all ages, including older ones, is based on humanitarian and professional responsibility, as well as personal human decency. At the same time, there also exist sound reasons to care about the welfare of older people, in particular, because their potential is essential for social development, especially when it comes to dealing with crises and disasters. However, older people are often seen as the unproductive ballast because of the increasing expenditure on their social and health needs. This is the economic backbone of ageism; overcoming it is necessary in order not to lose the development potential of aging societies.

In an aging society, the relative number of people of "productive" age inevitably decreases, with a corresponding increase in the proportion of people of retirement age and in late life, which are traditionally considered as "unproductive." As society ages, the labor force of young people is gradually reduced and, thus, the "first demographic dividend" of economic development is lost. However, in the later stages of demographic transition, the "second demographic dividend" forms in the aging society [26] due to an increase in life expectancy in general and in healthy life without sickness or disability. In this way, society gets an additional resource of qualified citizens for its economic and social development.

It should be noted that the second demographic dividend of an aging society, as well as the first dividend of a younger society, does not materialize automatically. In order for the potential of the demographic dividends to be realized, governmental policy should be balanced and implemented in proper time [35]. For example, measures that promote active and healthy aging are required in aging societies in order to involve older citizens in productive activities, whether in unpaid volunteering or the formal labor market. This refers to the prophylaxis of chronic diseases, appropriate treatment, and rehabilitation in health care, lifelong learning, and pension reform. 
Older people, like people of any other age, do not form a homogeneous group; they differ with respect to their health state, economic status, preferences, and ambitions. Many of today's older citizens take personal responsibility for their well-being and continue to contribute to the development of their communities, villages, towns, and society as a whole. They are the ones who suffer the greatest losses, including emotional distress, from the "measures directed to maintain the self-isolation regime for persons over 65 years of age." At the same time, the participation of these people, who became free from the guardianship of state paternalism and who possess knowledge and experience in getting over the trials and troubles of life, will be particularly valuable in overcoming the consequences of pandemics.

At the end of scientific articles, it is customary to declare whether the authors have a conflict of interest. The authors feel obliged to state that they belong to the $65+$ group and therefore cannot be completely impartial as they represent a significant part of the population with their unique features and specific needs. This population group, no less than that of people of any other age, has a full and equal right to a high quality of life that is accessible even under extreme conditions. The privilege of joining this group with time is not excluded for anyone. For those who are now in charge of making decisions about "people over 65 years of age," it is time to realize that, now, unlike in the Middle Ages, 65+ is a personal future for almost everyone. In this foreseeable future, SARS-CoV-2, unlike variola virus and poliovirus, is unlikely to leave humanity in peace. What is the future for us (you) then?

\section{COMPLIANCE WITH ETHICAL STANDARDS}

No experimentations involving animals or human were performed by any of the authors.

Conflicts of interest. The authors declare no conflict of interest.

\section{REFERENCES}

1. Golubev, A.G., Where does the Preston curve lead us? Adv. Gerontol., 2019, vol. 9, no. 2, pp. 115-125.

2. Golubev, A.G., Why and how do we age? A single answer to two questions, Adv. Gerontol., 2019, vol. 9, no. 1 , pp. 1-14.

3. Drapkina, O.M., Samorodskaya, I.V., Sivtseva, M.G., et al., COVID-19: urgent questions for estimating morbidity, prevalence, case fatality rates and mortality rates, Prof. Med., 2020, vol. 23, no. 1, pp. 7-13.

4. Appleby, J., What is happening to non-COVID deaths? Br. Med. J., 2020, vol. 369. https://doi.org/10.1136/bmj.m1607

5. Boreskie, K.F., Boreskie, P.E., and Melady, D., Age is just a number- and so is frailty: strategies to inform resource allocation during the COVID-19 pandemic, Can. J. Emerg. Med., 2020 vol. 22, no. 4, pp. 411-413. https://doi.org/10.1017/cem.2020.358
6. Box, G.E.P. and Draper, N.R., Empirical Model Building and Response Surfaces, New York, NY: Wiley, 1987.

7. Butler, R.N., Age-ism: another form of bigotry, Gerontologist, 1969, vol. 9, no. 4, pp. 243-246.

8. Celis-Morales, P.A., Gray, S., Petermann, F., et al., Walking pace is associated with lower risk of all-cause and cause-specific mortality, Med. Sci. Sports Exercise, 2019, vol. 51, no. 3, pp. 472- 480.

9. Czeisler, M.É., Howard, M.E., Robbins, R., et al., COVID-19: public compliance with and public support for stay-at-home mitigation strategies, medRxiv, 2020. https://doi.org/10.1101/2020.04.22.20076141

10. De Souto Barreto, P., Demougeot, L., Vellas, B., and Rolland, Y., Exercise training for preventing dementia, mild cognitive impairment, and clinically meaningful cognitive decline: a systematic review and meta-analysis, J. Gerontol., A, 2017, vol. 73, pp. 1504-1511.

11. Doi, A., Iwata, K., Kuroda, H., Hasuike, V., et al., Estimation of seroprevalence of novel coronavirus disease (COVID-19) using preserved serum at an outpatient setting in Kobe, Japan: a cross-sectional study, medRxiv, 2020.

https://doi.org/10.1101/2020.04.26.20079822

12. Drucker, D.J., Coronavirus infections and type 2 diabetes-shared pathways with therapeutic implications, Endocrinol. Rev., 2020, vol. 41, no. 3.

https://doi.org/10.1210/endrev/bnaa011

13. Emanuel, E.J., Persad, G., Upshur, R., et al., Fair allocation of scarce medical resources in the time of COVID-19, New Engl. J. Med., 2020.

https://doi.org/10.1056/NEJMsb2005114

14. Golubev, A., How could the Gompertz-Makeham law evolve, J. Theor. Biol., 2009, vol. 258, pp. 1-17.

15. Golubev, A., A 2D analysis of correlations between the parameters of the Gompertz-Makeham model (or law?) of relationships between aging, mortality, and longevity, Biogerontology, 2019, vol. 20, pp. 799-821.

16. Golubev, A.G., Commentary: Is life extension today a Faustian bargain?, Front. Med., 2018, vol. 5, no. 73 . https://doi.org/10.3389/fmed.2018.00073

17. Golubev, A.G. and Anisimov, V.N., Aging and cancer: Is glucose a mediator between them?, Oncotarget, 2019, vol. 10 , pp. $6758-6767$.

https://doi.org/10.18632/oncotarget.27344

18. Griffin, M.R., Zhu, Y., Moore, M.R., et al., U.S. hospitalizations for pneumonia after a decade of pneumococcal vaccination, New Engl. J. Med., 2013, vol. 369, pp. 155-163.

19. Huang, I., Lim, M.A., and Pranata, R., Diabetes mellitus is associated with increased mortality and severity of disease in COVID-19 pneumonia- $A$ systematic review, meta-analysis, and meta-regression, Diabetes Metab. Syndrome, 2020, vol. 14, pp. 395-403.

20. James, P., Kim, E. S., Kubzansky, L. D., et al., Optimism and healthy aging in women, Am. J. Prevent. Med., 2019, vol. 56, pp. 116-124.

21. Kikusui, T., How does social enrichment produce health benefits?, eLife, 2018, vol. 7. https://doi.org/10.7554/eLife.43666

22. Kissler, S.M., Tedijanto, P., Goldstein, E., et al., Projecting the transmission dynamics of SARS-CoV-2 
through the postpandemic period, Science, 2020. https://doi.org/10.1126/science.abb5793

23. Koff, W.P. and Williams, M.A., Covid-19 and immunity in aging populations $-A$ new research agenda, New Engl. J. Med., 2020. https://doi.org/10.1056/NEJMp2006761

24. Lee, D.-C., Brellenthin, A.G., Thompson, P.D., et al., Running as a key lifestyle medicine for longevity, Progr. Cardiovasc. Dis., 2017, vol. 60, pp. 45-55.

25. Marques, S., Mariano, J., Mendonça, J., et al., Determinants of ageism against older adults: a systematic review, Int. J. Environ. Res. Publ. Health, 2020, vol. 17, no. 7 , p. 2560. https://doi.org/10.3390/ijerph17072560

26. Mason, A., Lee, R., and Jiang, J.X., Demographic dividends, human capital, and saving, J. Econ. Ageing, 2016, vol. 7, pp. 106-122.

27. Nikolich-Žugich, J., The twilight of immunity: emerging concepts in aging of the immune system, Nat. Immunol., 2018, vol. 19, pp. 10-19.

28. Palmer, S., Albergante, L., Blackburn, P.P., and Newman, V.J., Thymic involution and rising disease incidence with age, Proc. Natl. Acad. Sci. U.S.A., 2018, vol. 115, pp. 1883-1888.

29. Roche, B., Garchitorena, A., and Roiz, D., Optimal strategies for quarantine stopping in France. General expected patterns of strategies focusing on contact between age groups, medRxiv, 2020.

https://doi.org/10.1101/2020.04.21.20073932

30. Salje, H., Tran Kiem, P., Lefrancq, N., et al., Estimating the burden of SARS-CoV-2 in France, Science, 2020. https://doi.org/10.1126/science.abc3517

31. Santesmasses, D., Castro, J.P., Zenin, A.A., et al., COVID-19 is an emergent disease of aging, medRxiv, 2020. https://doi.org/10.1101/2020.04.15.20060095

32. Santini, Z.I., Jose, P.E., Koyanagi, A., et al., Formal social participation protects physical health through enhanced mental health: a longitudinal mediation analysis using three consecutive waves of the Survey of Health, Ageing and Retirement in Europe (SHARE), Soc. Sci. Med., 2020, vol. 251. https://doi.org/10.1016/j.socscimed.2020.112906

33. Shakiba, M., Hashemi Nazari, S.S., Mehrabian, F., et al., Seroprevalence of COVID-19 virus infection in Guilan province, Iran, medRxiv, 2020. https://doi.org/10.1101/2020.04.26.20079244

34. Shamshirian, A., Heydari, K., Alizadeh-Navaei, R., et al., Cardiovascular diseases and COVID-19 mortality and intensive care unit admission: a systematic review and meta-analysis, medRxiv, 2020. https://doi.org/10.1101/2020.04.12.20062869

35. Sidorenko, A., Adjusting to population ageing: evidence for policy action, Popul. Horiz., 2015, vol. 12, pp. 1746-1081. https://doi.org/10.1515/pophzn-2015-0008

36. Stefan, N., Birkenfeld, A.L., Schulze, M.B., and Ludwig, D.S., Obesity and impaired metabolic health in patients with COVID-19, Nat. Rev. Endocrinol., 2020. https://doi.org/10.1038/s41574-020-0364-6

37. Thune, I. and Furberg, A.S., Physical activity and cancer risk: dose-response and cancer, all sites and sitespecific, Med. Sci. Sports Exercise, 2001, vol. 33, no. 6, pp. S530-S550.

38. Policy brief: the impact of COVID-19 on older persons, United Nations, 2020. https://unsdg.un.org/sites/default/files/2020-05/Policy-Brief-The-Impact-of-COVID19-on-Older-Persons.pdf.

39. Yang, Y.P., Boen, P., Gerken, K., et al., Social relationships and physiological determinants of longevity across the human life span, Proc. Natl. Acad. Sci. U.S.A., 2016, vol. 113, pp. 578-583.

40. Zhu, L., She, Z.-G., Cheng, X., et al., Association of blood glucose control and outcomes in patients with COVID-19 and pre-existing type 2 diabetes, Cell Metab., 2020.

https://doi.org/10.1016/j.cmet.2020.04.021

Translated by E. Martynova 